\section{Treatment of dystocia in a leopard gecko (Eublepharis macularius) by percutaneous ovocentesis}

\author{
A. J. Hall, G. A. LeWbart
}

DYSTOCIA is a relatively common problem reported in captive reptiles; it is more frequently reported in snakes and turtles than in lizards (Lloyd 1990, DeNardo 1996). The first approach to medical treatment of the condition is often to stimulate contractions of the oviduct using oxytocin, which is generally highly effective in turtles (Peters and Coote 1977, DeNardo 1996). The synthetic hormone arginine vasotocin has been considered as an alternative to oxytocin, although its clinical usefulness has been limited by the poor availability of medical-grade formulations (DeNardo 1996, Carpenter and others 2001). In larger lizards, such as green iguanas (Iguana iguana), cases of dystocia refractory to oxytocin generally require salpingotomy or ovariosalpingectomy if there is no intention of breeding the lizard in the future (Frye 1991, DeNardo 1996). For smaller lizards, surgical treatment can be a challenging procedure, requiring magnification and microsurgical expertise. In addition, surgery may carry a significant risk to the animal, since dystocic lizards are generally in poor metabolic condition (DeNardo 1996). For cases of dystocia that prove refractory to medical treatment, a second treatment option, used in snakes and turtles, is to aspirate the yolk of retained eggs, thereby reducing their size and facilitating natural oviposition (DeNardo 1996). This short communication describes the treatment of refractory dystocia in a young leopard gecko (Eublepharis macularius) by percutaneous ovocentesis, a procedure apparently previously unreported in lizards.

A 15-month-old female leopard gecko was presented with a history of decreased appetite and decreased body condition; the owner believed it to have been 'egg-bound' for over one week. The gecko had been captive-bred from a known breeding stock by a local herptile hobbyist. The owner reported that the animal had once previously had a prolonged period of being egg-bound, which eventually resolved without treatment. The gecko was housed alone in a plastic container with a lid to retain moisture, a spot lamp providing heat, and newspaper substrate. The owner reported that the animal was fed a diet of primarily crickets, with a commercial calcium supplement. The current episode of dystocia was described as being more prolonged than the previous incident, and the gecko was now showing signs of inappetence and cachexia. The owner also reported that the gecko had never been housed with a male, indicating that the eggs were not fertile. Upon presentation, the gecko's weight was $43 \mathrm{~g}$ and it was bright, alert and responsive. Physical examination revealed significant abdominal distention, although palpation did not appear to elicit any discomfort. Two large, oblong structures were visible through the skin using transillumination. The lizard was in fair body condition, with minimal fat reserves in the tail. No other abnormalities were noted.

A faecal sample was analysed for intestinal parasites by faecal flotation and direct smear, both of which were negative. Because blood analysis was felt to be of limited diagnostic use, it was decided initially to improve the animal's husbandry and provide an appropriate substrate (sand) to encourage ovi-
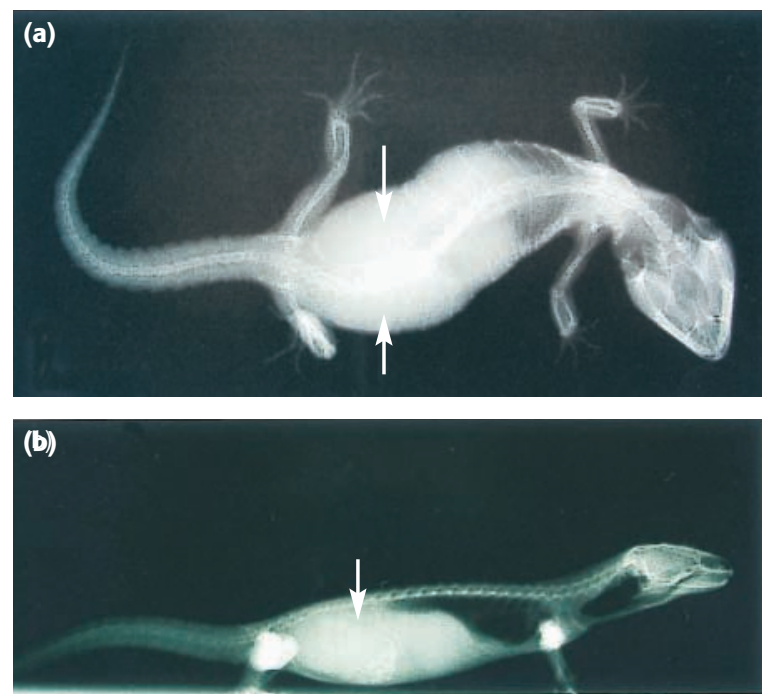

FIG 1 : (a) Dorsoventral and (b) left lateral radiographs of a 15-month-old female leopard gecko with two shelled eggs visible in the abdomen (arrows)

position. The gecko was provided a plastic container with a single opening, containing moistened vermiculite substrate, and kept in a terrarium in which the temperature ranged from 27 to $30^{\circ} \mathrm{C}$. The gecko was offered crickets and monitored for eating, drinking and defecation. The gecko was returned a week later, and the owner reported that the eggs had not passed and the animal was still not eating or defecating. Induction of parturition was attempted by administering 20 $\mathrm{mg} / \mathrm{kg}$ calcium gluconate intramuscularly, followed an hour later by two doses of $2 \mathrm{iu} / \mathrm{kg}$ oxytocin (Oxoject; Vetus Animal Health) given intramuscularly, one hour apart. The gecko was then returned to its terrarium for observation.

Thirty-six hours after the attempt to induce parturition, oviposition had still not occurred. Given the gecko's decreasing body condition, anorexia and continuing lack of defecation, it was decided to intervene further. Although the presence of eggs had already been established visually and by palpation, radiographs were taken to determine the exact number, size, and position of the eggs more accurately, since unusually large or malformed eggs could have made further oxytocin treatments risky. Dorsoventral and left lateral views (using a horizontal beam) were obtained, and revealed two oblong radio-opaque structures, consistent in size, shape, and location with shelled eggs (Fig 1). The radiographs also revealed cranial displacement of the abdominal viscera and encroachment on the caudal lung field. These findings, along with the history of anorexia and constipation, indicated a more aggressive form of treatment. Surgical intervention by ovariosalpingectomy was considered but decided against due to the expense and the risk of prolonged anaesthesia. It was decided to attempt percutaneous ovocentesis, to reduce the size of the eggs and thus make them easier for the gecko to pass.

The gecko was anaesthetised by chamber induction with 4 per cent isoflurane in oxygen until a light plane of anaesthesia was achieved (approximately five minutes). It was then removed from the chamber and held upright while the aspiration site was prepared using 10 per cent topical povidoneiodine solution. The location for aspiration was determined by visibly identifying the middle of the egg craniocaudally and at an approximately $45^{\circ}$ angle between the ventral and lateral midlines. A sterile $23 \mathrm{G}$ butterfly catheter (Terumo Surglo Winged Infusion Set, $0.65 \times 19 \mathrm{~mm}$ ) was inserted approximately $2 \mathrm{~cm}$ through the skin and into the egg (Fig 2a). Using a $6 \mathrm{ml}$ syringe, the egg was aspirated using gentle negative 

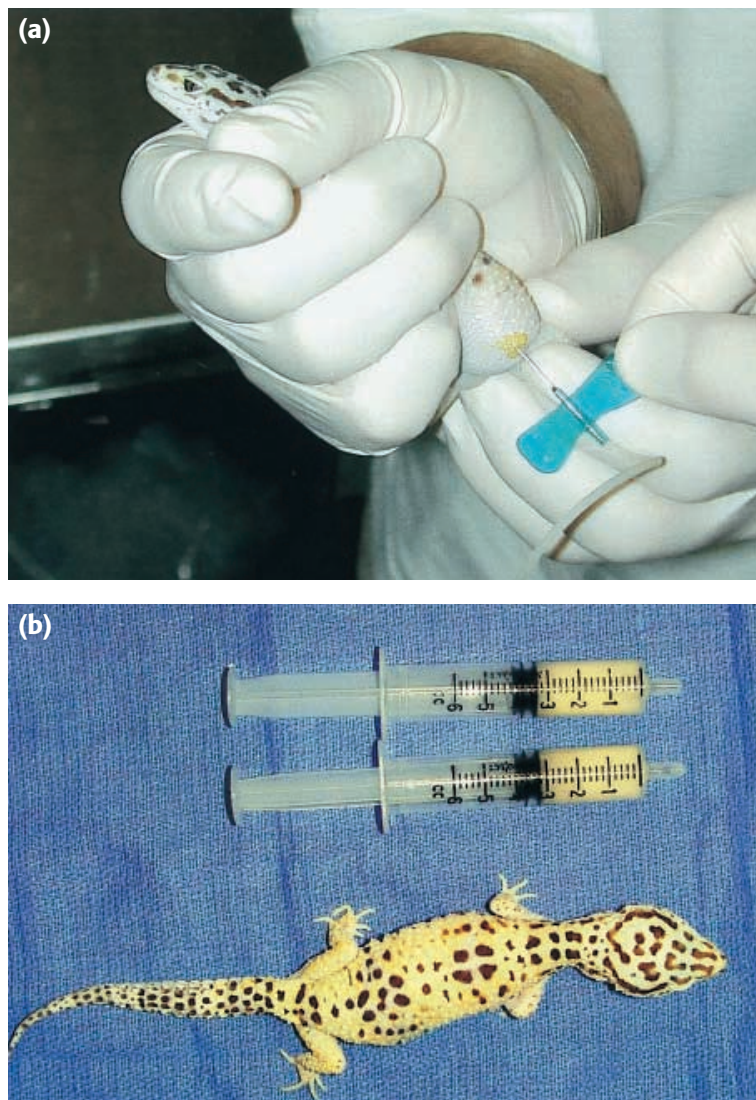

FIG 2: (a) Percutaneous ovocentesis of a 15-month-old leopard gecko; (b) appearance of the gecko after the procedure, and syringes containing the yolk aspirated from each side of the animal

pressure and $3.2 \mathrm{ml}$ of opaque, viscous yolk was removed (Fig $2 \mathrm{~b})$. The procedure was repeated on the other side, from which $3.4 \mathrm{ml}$ of yolk was removed. Following the procedure the abdominal distention was visibly reduced. The gecko was returned to its terrarium to recover.

The gecko fully recovered from the brief period of anaesthesia within 30 minutes, and was observed for passage of the eggs. Within 36 hours of the ovocentesis procedure, two shrivelled, shrunken eggs were found in the terrarium (Fig 3). The gecko regained its appetite, and returned to a healthy body condition over the following month, during which it was fed a diet of nutritionally enhanced mealworms and crickets. The gecko developed significant fat stores in its tail, continued to maintain a normal appetite, and produced faeces and urates regularly. At a re-examination two months after the procedure, the gecko's weight was $65 \mathrm{~g}$, and physical examination revealed no abnormalities (Fig 4).

In this case, percutaneous ovocentesis effectively resolved the dystocia and enabled the gecko to pass the eggs. Potential risks associated with this procedure include leakage of yolk

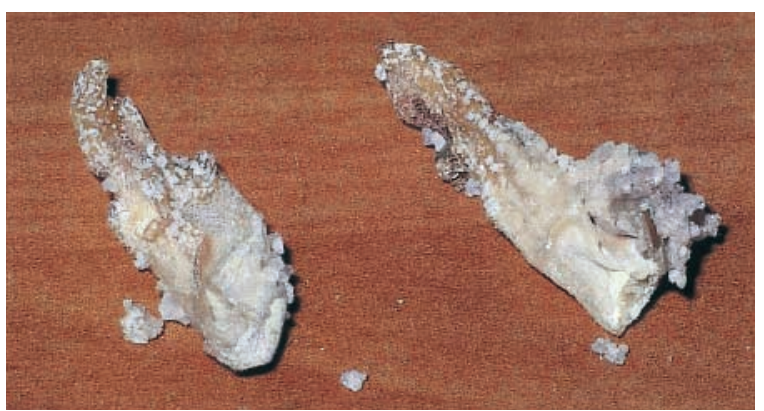

FIG 3: Eggs, covered in sand from the terrarium, passed by the leopard gecko less than $\mathbf{3 6}$ hours after the percutaneous ovocentesis procedure

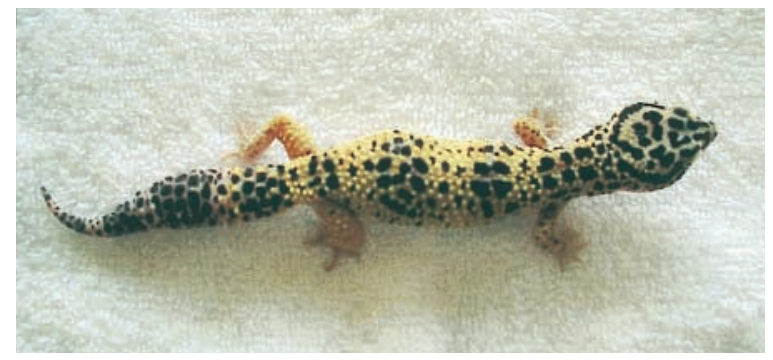

FIG 4: Two months after the procedure, the gecko has returned to a healthy body condition

into the coelomic cavity, accidental aspiration of the viscera, and possible coelomitis, but none occurred in the present case. It is also important to note that the procedure provides only a temporary resolution of dystocia, whereas ovariosalpingectomy would prevent its recurrence. Nonetheless, percutaneous ovocentesis provides a simple, safe, and inexpensive alternative to surgery for treating dystocia in geckos, particularly in cases that are refractory to medical treatment.

\section{ACKNOWLEDGEMENTS}

The authors thank Daniel Dombrowski and Deborah Corpuz for assisting in the management of this case, and Larry S. Christian for taking photographs of the procedure and technical assistance. They are also grateful to Craig Harms for his editorial comments and review of this article.

\section{References}

CARPENTER, J. W., MASHIMA, T. Y. \& RUPIPER, D. J. (2001)Reptiles. In Exotic Animal Formulary. 2nd edn. Philadelphia, W. B. Saunders. pp 7072

DENARDO, D. (1996) Dystocia. In Reptile Medicine and Surgery. Ed D. R. Mader. Philadelphia, W. B. Saunders. pp 370-374

FRYE, F. L. (1991) Reproduction in reptiles. In Reptile Care: An Atlas of Diseases and Treatments. Ed F. L. Frye. Neptune City, TFH Publications. p 376

LLOYD, M. L. (1990) Reptilian dystocias review: causes, prevention, management and comments on the synthetic hormone vasotocin. Proceedings of the American Association of Zoo Veterinarians, 1990. pp 290-296

PETERS, A. R. \& COOTE, J. (1977) Dystocia in a snake. Veterinary Record 100, 